\title{
Weak Convergence for a Class of Stochastic Fractional Equations Driven by Fractional Noise
}

\author{
Xichao Sun ${ }^{1}$ and Junfeng Liu ${ }^{2}$ \\ ${ }^{1}$ Department of Mathematics and Physics, Bengbu College, 1866 Caoshan Road, Bengbu, Anhui 233030, China \\ ${ }^{2}$ School of Mathematics and Statistics, Nanjing Audit University, 86 West Yu Shan Road, Pukou, Nanjing 211815, China \\ Correspondence should be addressed to Xichao Sun; sunxichao626@126.com
}

Received 21 September 2013; Revised 27 December 2013; Accepted 27 December 2013; Published 12 February 2014

Academic Editor: Ming Li

Copyright (c) 2014 X. Sun and J. Liu. This is an open access article distributed under the Creative Commons Attribution License, which permits unrestricted use, distribution, and reproduction in any medium, provided the original work is properly cited.

\begin{abstract}
We consider a class of stochastic fractional equations driven by fractional noise on $(t, x) \in[0, T] \times[0,1] \partial u / \partial t=D_{\delta}^{\alpha} u+f(t, x, u)+$ $\partial^{2} B^{H}(t, x) / \partial t \partial x$, with Dirichlet boundary conditions. We formally replace the random perturbation by a family of sequences based on Kac-Stroock processes in the plane, which approximate the fractional noise in some sense. Under some conditions, we show that the real-valued mild solution of the stochastic fractional heat equation perturbed by this family of noises converges in law, in the space $\mathscr{C}([0, T] \times[0,1])$ of continuous functions, to the solution of the stochastic fractional heat equation driven by fractional noise.
\end{abstract}

\section{Introduction}

In recent years, there has been considerable interest in studying fractional equations due to interesting properties and applications in various scientific areas including image analysis, risk management, and statistical mechanics (see Droniou and Imbert [1] and Uchaikin and Zolotarev [2] for a survey of applications). Much effort has been devoted to apply the fractional calculus to mathematical problems in science and engineering. For example, Chen et al. [3] and Li et al. [4] studied the fractional-order networks and $\mathrm{Li}$ [5] investigated fractal time series. More works on the fields can be found in [6-12] and the references therein. Stochastic partial differential equation involving fractional Laplacian operator (which is an integrodifferential operator) has been studied by many authors. For example, Mueller [13] and $\mathrm{Wu}[14]$ proved the existence of a solution of stochastic fractional heat and Burgers equation perturbed by a stable noise, respectively. Other related references are Chang and Lee [15], Truman and $\mathrm{Wu}$ [16], Liu et al. [17], Wu [18], and the references therein.

On the other hand, weak convergence to Brownian motion, fractional Brownian motion, and related stochastic processes have been considered extensively since the work of Taqqu [19] and Delgado and Jolis [20]. Recently, many researchers are interested in studying weak convergence of stochastic differential equation. Some surveys could be found in Bardina et al. [21], Boufoussi and Hajji [22], and Mellall and Ouknine [23]. Bardina et al. [21] studied the convergence in law, in the space $\mathscr{C}([0, t] \times[0,1])$ of continuous functions, of the solution of

$$
\frac{\partial X_{n}}{\partial t}(t, x)-\frac{\partial^{2} X_{n}}{\partial x^{2}}(t, x)=\theta_{n}(t, x),
$$

with vanishing initial data and Dirichlet boundary conditions, towards the solution of

$$
\frac{\partial X}{\partial t}(t, x)-\frac{\partial^{2} X}{\partial x^{2}}(t, x)=\dot{W}(t, x),
$$

where $(t, x) \in[0, T] \times[0,1]$ and $\theta_{n}$ is a noisy input which converges to white noise $\dot{W}$. Mellall and Ouknine [23] considered the quasilinear stochastic heat equation on $[0,1]$

$$
\frac{\partial u}{\partial t}(t, x)-\frac{\partial^{2} u}{\partial x^{2}}(t, x)=b(u(t, x))+\frac{\partial^{2} B^{H}(t, x)}{\partial t \partial x},
$$

with Dirichlet boundary conditions

$$
u(t, 0)=u(t, 1)=0, \quad t \in[0, T],
$$


and initial condition $u(0, x)=u_{0}(x), x \in[0,1]$, where $\partial^{2} B^{H}(t, x) / \partial t \partial x$ is a fractional noise with Hurst parameter $H \in(1 / 2,1)$.

Motivated by these works, we consider the weak convergence for the following stochastic fractional heat equation driven by fractional noise on $(t, x) \in[0, T] \times[0,1]$ :

$$
\begin{gathered}
\frac{\partial u}{\partial t}=D_{\delta}^{\alpha} u+f(t, x, u)+\frac{\partial^{2} B^{H}(t, x)}{\partial t \partial x}, \\
u(t, 0)=u(t, 1)=0
\end{gathered}
$$

where $D_{\delta}^{\alpha}$ is the fractional Laplacian operator with respect to the spatial variable, to be defined in Section 2 which was recently introduced by Debbi [24] and Debbi and Dozzi [25], and $B^{H}(t, x)$ is a fractional noise on $[0, T] \times[0,1]$ with Hurst index $H>1 / 2$ defined on a complete probability space $\left\{\Omega, \mathscr{F},\left\{\mathscr{F}_{t}\right\}_{t \geq 0}, P\right\}$. Actually, we understand (5) in the sense of Walsh [26], and so one can present a mild formulation of (5) as follows:

$$
\begin{aligned}
u(t, x)= & \int_{0}^{1} G_{\alpha, \delta}(t, x-y) u_{0}(y) d y \\
& +\int_{0}^{t} \int_{0}^{1} G_{\alpha, \delta}(t-s, x-y) f(s, y, u(s, y)) d y d s \\
& +\int_{0}^{t} \int_{0}^{1} G_{\alpha, \delta}(t-s, x-y) B^{H}(d s, d y)
\end{aligned}
$$

where $G_{\alpha, \delta}(\cdot, *)$ denotes the Green function associated with (5).

The rest of this paper is organized as follows. In Section 2, we begin by making some notation and by recalling some basic preliminaries which will be needed later. In Section 3, we will prove weak limit theorems for (5) in space $\mathscr{C}([0, t] \times$ $[0,1])$. Most of the estimates of this paper contain unspecified constants. An unspecified positive and finite constant will be denoted by $C$, which may not be the same in each occurrence. Sometimes we will emphasize the dependence of these constants upon parameters.

\section{Preliminaries}

In this section, we briefly recall some basic definitions of fractional noise and Green function.

2.1. Fractional Noise. For each $t \in[0, T]$, let $\mathscr{F}_{t}^{H}$ be the $\sigma$-field generated by the random variables $\left\{B^{H}(t, A), t \in\right.$ $[0, T], A \in \mathscr{B}[0,1]\}$ and the sets of probability zero, and denote by $\mathscr{P}$ the $\sigma$-field of progressively measurable subsets of $[0, T] \times \Omega$.

We denote by $\mathscr{E}$ the set of step functions on $[0, T] \times[0,1]$. Let $\mathscr{H}$ be the Hilbert space defined as the closure of $\mathscr{E}$ with respect to the scalar product

$$
\left\langle\mathbb{1}_{[0, t] \times A}, \mathbb{1}_{[0, s] \times B}\right\rangle_{\mathscr{H}}=|A \cap B| R_{H}(t, s),
$$

where covariance kernel $R_{H}(t, s)=(1 / 2)\left[t^{2 H}+s^{2 H}-|t-s|^{2 H}\right]$ and $|A|$ denotes the Lebesgue measure of the set $A$.
According to Nualart and Ouknine [27], the mapping $\mathbb{1}_{[0, t] \times A} \rightarrow B^{H}(t, A)$ can be extended to an isometry between $\mathscr{H}$ and the Gaussian space $H_{1}\left(B^{H}\right)$ associated with $B^{H}$ and denoted by

$$
\varphi \longmapsto B^{H}(\varphi):=\int_{[0, t] \times A} \varphi(s, y) B^{H}(d s, d y)
$$

Define the linear operator $K_{H}^{*}: \mathscr{E} \mapsto L^{2}([0, T])$ by

$$
\begin{aligned}
K_{H}^{*}(\varphi)= & K_{H}(T, s) \varphi(s, x) \\
& +\int_{s}^{T}(\varphi(r, x)-\varphi(s, x)) \frac{\partial K_{H}}{\partial r}(r, s) d r,
\end{aligned}
$$

where $K_{H}$ is the square integrable kernel given by

$$
\begin{aligned}
& K_{H}(t, s) \\
& := \begin{cases}c_{H}(t-s)^{H-1 / 2}+C_{H} s^{1 / 2-H} \\
\quad \times \int_{s}^{t}(u-s)^{H-3 / 2}\left(1-\left(\frac{s}{u}\right)^{1 / 2-H}\right) d u, & 0<s \leq t, \\
0, & \text { otherwise, }\end{cases}
\end{aligned}
$$

with $c_{H}=(2 H \Gamma(3 / 2-H) / \Gamma(H+1 / 2) \Gamma(2-2 H))^{1 / 2}$, and one can get

$$
\frac{\partial K_{H}}{\partial t}(t, s)=c_{H}\left(\frac{1}{2}-H\right)\left(\frac{t}{s}\right)^{H-1 / 2}(t-s)^{H-3 / 2} .
$$

Moreover, the kernel $K_{H}$ satisfies the following property:

$$
\int_{0}^{s \wedge t} K_{H}(t, r) K_{H}(s, r) d r=R_{H}(t, s)
$$

$R_{H}(t, s)$ being the covariance kernel of the fractional Brownian motion. Then, for any pair of step functions $\varphi$ and $\psi$ in $\mathscr{E}$ we have

$$
\left\langle K_{H}^{*}(\varphi), K_{H}^{*}(\psi)\right\rangle_{L^{2}([0, T] \times[0,1])}=\langle\varphi, \psi\rangle_{\mathscr{H}},
$$

because

$$
\left(K_{H}^{*} 1_{[0, t] \times A}\right)(s, x)=K_{H}(t, s) 1_{[0, t] \times A}(s, x) .
$$

As a consequence, the operator $K_{H}^{*}$ provides an isometry between the Hilbert space $\mathscr{H}$ and $L^{2}([0, T] \times[0,1])$. Hence, the Gaussian family $\{W(t, A), t \in[0, T], A \in \mathscr{B}[0,1]\}$ defined by

$$
W(t, A)=B^{H}\left(\left(K_{H}^{*}\right)^{-1}\left(1_{[0, t] \times A}\right)\right)
$$

is a space-time white noise, and the process $B^{H}$ has an integral representation of the form

$$
B^{H}(t, x)=\int_{0}^{t} \int_{0}^{x} K_{H}(t, s) W(d s, d y) .
$$


Now, we can present a mild formulation of (5) as follows:

$$
\begin{aligned}
u(t, x)= & \int_{0}^{1} G_{\alpha, \delta}(t, x-y) u_{0}(y) d y \\
& +\int_{0}^{t} \int_{0}^{1} G_{\alpha, \delta}(t-s, x-y) f(s, y, u(s, y)) d y d s \\
& +\int_{0}^{t} \int_{0}^{1} K_{H}^{*} G_{\alpha, \delta}(t-s, x-y) W(d s, d y) .
\end{aligned}
$$

That is, the last term of (6) is equal to

$$
\begin{aligned}
& \int_{0}^{t} \int_{0}^{1} G_{\alpha, \delta}(t-s, x-y) B^{H}(d s, d y) \\
& \quad=\int_{0}^{t} \int_{0}^{1} K_{H}^{*} G_{\alpha, \delta}(t-s, x-y) W(d s, d y) .
\end{aligned}
$$

2.2. Green Function. In this subsection, we will introduce the nonlocal factional differential operator $D_{\delta}^{\alpha}$ defined via its Fourier transform $\mathscr{F}$ by

$$
\mathscr{F}\left(D_{\delta}^{\alpha} \varphi\right)(\xi)=-|\xi|^{\alpha} \exp \left(-i \delta \frac{\pi}{2} \operatorname{sgn}(\xi)\right) \mathscr{F}(\varphi)(\xi)
$$

In this paper, we will assume that $|\delta| \leq \min \left\{\alpha-[\alpha]_{2}, 2+[\alpha]_{2}-\right.$ $\alpha\}, i=1, \ldots, d,[\alpha]_{2}$, is the largest even integer less or equal to $\alpha$ (even part of $\alpha$ ), and $\delta \in 2 \mathbb{N}+1$.

The operator $D_{\delta}^{\alpha}$ is a closed, densely defined operator on $L^{2}(\mathbb{R})$ and it is the infinitesimal generator of a semigroup which is in general not symmetric and not a contraction. This operator is a generalization of various well-known operators, such as the Laplacian operator (when $\alpha=2$ ), the inverse of the generalized Riesz-Feller potential (when $\alpha>2$ ), and the Riemann-Liouville differential operator (when $|\delta|=2+[\alpha]_{2}$ or $|\delta|=\alpha-[\alpha])$. It is self-adjoint only when $\delta=0$ and, in this case, it coincides with the fractional power of the Laplacian. We refer the readers to Debbi [24], Debbi and Dozzi [25], and Komatsu [28] for more details about this operator.

According to Komatsu [28], $D_{\delta}^{\alpha}$ can be represented for $1<$ $\alpha<2$ by

$$
\begin{aligned}
D_{\delta}^{\alpha} \varphi(x)=\int_{\mathbb{R}} & \frac{\varphi(x+y)-\varphi(x)-y \varphi^{\prime}(x)}{|y|^{1+\alpha}} \\
& \times\left(\kappa_{-}^{\delta} 1_{(-\infty, 0)}(y)+\kappa_{+}^{\delta} 1_{(-0,+\infty)}(y)\right) d y
\end{aligned}
$$

and for $0<\alpha<1$ by

$$
\begin{aligned}
D_{\delta}^{\alpha} \varphi(x)=\int_{\mathbb{R}} \frac{\varphi(x+y)-\varphi(x)}{|y|^{1+\alpha}} \\
\quad \times\left(\kappa_{-}^{\delta} 1_{(-\infty, 0)}(y)+\kappa_{+}^{\delta} 1_{(-0,+\infty)}(y)\right) d y,
\end{aligned}
$$

where $\kappa_{-}^{\delta}$ and $\kappa_{+}^{\delta}$ are two nonnegative constants satisfying $\kappa_{-}^{\delta}+$ $\kappa_{+}^{\delta}>0$ and $\varphi$ is a smooth function for which the integral exists, and $\varphi^{\prime}$ is its derivative. This representation identifies it as the infinitesimal generator for a nonsymmetric $\alpha$-stable Lévy process.

Let $G_{\alpha, \delta}(t, x)$ be the fundamental solution of the following Cauchy problem:

$$
\begin{gathered}
\frac{\partial u}{\partial t}(t, x)=D_{\delta}^{\alpha}(t, x), \\
u(0, x)=\delta_{0}(x), \quad t>0, x \in \mathbb{R},
\end{gathered}
$$

where $\delta_{0}(\cdot)$ is the Dirac distribution. By Fourier transform, we see that $G_{\alpha, \delta}(t, x)$ is given by

$$
\begin{aligned}
& G_{\alpha, \delta}(t, x) \\
& \quad=\frac{1}{2 \pi} \int_{\mathbb{R}} \exp \left(-i z x-t|z|^{\alpha} \exp \left(-i \delta \frac{\pi}{2} \operatorname{sgn}(z)\right)\right) d z .
\end{aligned}
$$

The relevant parameters $\alpha$, called the index of stability, and $\delta$ (related to the asymmetry), improperly referred to as the skewness, are real numbers satisfying $|\delta| \leq \min \left\{\alpha-[\alpha]_{2}, 2+\right.$ $\left.[\alpha]_{2}-\alpha\right\}$, and $\delta=0$ when $\delta \in 2 \mathbb{N}+1$.

Let us list some known facts on $G_{\alpha, \delta}(t, x)$ which will be used later on (see, e.g., Debbi [24] and Debbi and Dozzi [25]).

Lemma 1. Let $\alpha \in(0, \infty) /\{\mathbb{N}\}$; one has the following:

(1) the function $G_{\alpha, \delta}(t, \cdot)$ is not in general symmetric relatively to $x$ and it is not everywhere positive;

(2) for any $s, t \in(0, \infty)$ and $x \in \mathbb{R}$,

$$
\frac{\partial^{n}}{\partial x^{n}} G_{\alpha, \delta}(t, x)=(s)^{-(n+1) / \alpha} G_{\alpha, \delta}\left(s^{-1} t, s^{-1 / \alpha} x\right),
$$

or equivalently,

$\frac{\partial^{n}}{\partial x^{n}} G_{\alpha, \delta}(t, x)=(t)^{-(n+1) / \alpha} G_{\alpha, \delta}\left(1,(t)^{-1 / \alpha} x\right)$

(3) $G_{\alpha, \delta}(s, \cdot) * G_{\alpha, \delta}(t, \cdot)=G_{\alpha, \delta}(s+t, \cdot)$ for any $s, t \in(0, \infty)$;

(4) For $n \geq 1$, there exist some constants $C$ and $C_{n}>0$ such that, for all $x \in \mathbb{R}$,

$$
\begin{gathered}
\left|G_{\alpha, \delta}(1, x)\right| \leq C \frac{1}{1+|x|^{1+\alpha}}, \\
\left|\frac{\partial^{n}}{\partial x^{n}} G_{\alpha, \delta}(1, x)\right| \leq C_{n} \frac{|x|^{\alpha+n-1}}{\left(1+|x|^{\alpha+n}\right)^{2}}
\end{gathered}
$$

(5) $\int_{0}^{T} \int_{\mathbb{R}}\left|G_{\alpha, \delta}(t, x)\right|^{\lambda} d t d x<\infty$ if and only if $1 / \alpha<\lambda<$ $\alpha$. 


\section{Main Results and Its Proof}

Our aim is to prove that the mild solution of (5), given by (17), can be approximated in law in the space $\mathscr{C}([0, t] \times[0,1])$ by the processes

$$
\begin{aligned}
u_{n}(t, x)= & \int_{0}^{1} G_{\alpha, \delta}(t, x-y) u_{0}(y) d y \\
& +\int_{0}^{t} \int_{0}^{1} G_{\alpha, \delta}(t-s, x-y) f\left(s, y, u_{n}(s, y)\right) d y d s \\
& +\int_{0}^{t} \int_{0}^{1} K_{H}^{*} G_{\alpha, \delta}(t-s, x-y) \theta_{n}(s, y) d y d s,
\end{aligned}
$$

where $\left\{\theta_{n}(t, x)\right\}_{n \in \mathbb{N}}$ is a weak approximation of a Brownian sheet; that is, $\left\{\theta_{n}(t, x)\right\}_{n \in \mathbb{N}},(t, x) \in[0, T] \times[0,1]$ is a family of Kac-Stroock processes in the plane which is square integral a.s., defined by

$$
\theta_{n}(t, x)=n \sqrt{t x}(-1)^{N_{n}(t, x)}, \quad n \in \mathbb{N},
$$

$N_{n}(t, x)=N(\sqrt{n} t, \sqrt{n} x)$ and $\{N(t, x),(t, x) \in[0, T] \times[0,1]\}$ is a standard Poisson process in plane.

Theorem 2. Let $\left\{\theta_{n}(t, x),(t, x) \in[0, T] \times[0,1]\right\}, n \in \mathbb{N}$, be the Kac-Stroock processes in the plane. Assume that $u_{0}:[0,1] \rightarrow$ $\mathbb{R}$ is a continuous function and $f$ satisfies the following linear growth conditions:

$$
|f(t, x, u)| \leq C(1+|u|)
$$

and uniformly Lipschitz conditions

$$
|f(t, x, u)-f(t, x, u)| \leq C|u-v| .
$$

Then, the family of stochastic processes $\left\{u_{n}, n \in \mathbb{N}\right\}$ defined by (27) converges in law, as $n$ tends to infinity, in the space $\mathscr{C}([0, T] \times[0,1])$, to the mild solution $u$ of $(5)$, given by $(17)$.

In order to prove Theorem 2, we will focus on the linear problem, which is amount to establish the convergence in law, in the space $\mathscr{C}([0, T] \times[0,1])$, of the solutions of

$$
\frac{\partial X_{n}}{\partial t}-\frac{\partial^{2} X_{n}}{\partial x^{2}}=\frac{\partial^{2} B_{n}^{H}}{\partial t \partial x}
$$

with vanishing initial data and Dirichlet boundary conditions, toward the solution of

$$
\frac{\partial X}{\partial t}-\frac{\partial^{2} X}{\partial x^{2}}=\frac{\partial^{2} B^{H}}{\partial t \partial x}
$$

where the solutions of (31) and (32) are, respectively, given by

$$
\begin{gathered}
X_{n}(t, x)=\int_{0}^{t} \int_{0}^{1} K_{H}^{*} G_{\alpha, \delta}(t-s, x-y) \theta_{n}(s, y) d y d s, \\
X(t, x)=\int_{0}^{t} \int_{0}^{1} K_{H}^{*} G_{\alpha, \delta}(t-s, x-y) W(d s, d y) .
\end{gathered}
$$

In the following, we need two results which can be found in Bardina et al. [21]. The first one leads to the tightness in $\mathscr{C}([0, T] \times[0,1])$ of a family.
Lemma 3. Let $\left\{X_{n}, n \in \mathbb{N}\right\}$ be a family of random variables taking values in $\mathscr{C}([0, T] \times[0,1])$. The family of the laws of $\left\{X_{n}, n \in \mathbb{N}\right\}$ is tight, if there exist $p^{\prime}, p>0, \delta>2$, and $a$ constant $C$ such that

$$
\sup _{n \geq 1} E\left|X_{n}(0,0)\right|^{p^{\prime}}<\infty
$$

and, for every $t, t^{\prime} \in[0, T]$ and $x, x^{\prime} \in[0,1]$,

$$
\sup _{n \geq 1} E\left|X_{n}\left(t^{\prime}, x^{\prime}\right)-X_{n}(t, x)\right|^{p}<C\left(\left|x^{\prime}-x\right|+\left|t^{\prime}-t\right|\right)^{\delta} .
$$

The second one is a technical lemma.

Lemma 4. Denote by $\theta_{n}(t, x)$ the Kac-Stroock kernels; for any even $n \in \mathbb{N}$, there exists a constant $C_{n}$, such that, for any $t_{1}, t_{2} \in$ $[0, T]$ and $x_{1}, x_{2} \in[0,1]$ satisfying $0<t_{1}<t_{2}<2 t_{1}$ and $0<x_{1}<x_{2}<2 x_{1}$, one can get

$$
\begin{gathered}
\sup _{n \geq 1} E\left[\left(\int_{t_{1}}^{t_{2}} \int_{x_{1}}^{x_{2}} f(s, x) \theta_{n}(s, x) d x d s\right)^{n}\right] \\
\leq C_{n}\left(\int_{t_{1}}^{t_{2}} \int_{x_{1}}^{x_{2}} f^{2}(s, x) d x d s\right)^{n / 2},
\end{gathered}
$$

for any $f(s, x) \in L^{2}([0, T] \times[0,1])$.

Proposition 5. The family of processes $\left\{X_{n}, n \in \mathbb{N}\right\}$ is tight in $\mathscr{C}([0, T] \times[0,1])$.

Proof. We first estimate the moment of order $m$ of the quantity

$$
\begin{aligned}
X_{n}\left(t^{\prime}, x^{\prime}\right)-X_{n}(t, x) & =\int_{0}^{t^{\prime}} \int_{0}^{1} K_{H}^{*} G_{\alpha, \delta}\left(t^{\prime}-s, x^{\prime}-y\right) \theta_{n}(s, y) d y d s \\
& -\int_{0}^{t} \int_{0}^{1} K_{H}^{*} G_{\alpha, \delta}(t-s, x-y) \theta_{n}(s, y) d y d s,
\end{aligned}
$$

which is equal to

$$
\begin{gathered}
\int_{0}^{t} \int_{0}^{1} K_{H}^{*}\left(G_{\alpha, \delta}\left(t^{\prime}-s, x^{\prime}-y\right)\right. \\
\left.-G_{\alpha, \delta}\left(t^{\prime}-s, x-y\right)\right) \theta_{n}(s, y) d y d s \\
+\int_{0}^{t} \int_{0}^{1} K_{H}^{*}\left(G_{\alpha, \delta}\left(t^{\prime}-s, x-y\right)\right. \\
\left.\quad-G_{\alpha, \delta}(t-s, x-y)\right) \theta_{n}(s, y) d y d s \\
+\int_{t}^{t^{\prime}} \int_{0}^{1} K_{H}^{*} G_{\alpha, \delta}\left(t^{\prime}-s, x^{\prime}-y\right) \theta_{n}(s, y) d y d s .
\end{gathered}
$$


Advances in Mathematical Physics

5

By Lemma 4, one can get

$$
\begin{aligned}
& \sup _{n \geq 1} E\left|X_{n}\left(t^{\prime}, x^{\prime}\right)-X_{n}(t, x)\right|^{m} \\
& \leq C_{m}\left[\int _ { 0 } ^ { t } \int _ { 0 } ^ { 1 } \left[K _ { H } ^ { * } \left(G_{\alpha, \delta}\left(t^{\prime}-s, x^{\prime}-y\right)\right.\right.\right. \\
& \left.\left.\left.\quad-G_{\alpha, \delta}\left(t^{\prime}-s, x-y\right)\right)\right]^{2} d y d s\right]^{m / 2} \\
& +C_{m}\left[\int _ { 0 } ^ { t } \int _ { 0 } ^ { 1 } \left[K _ { H } ^ { * } \left(G_{\alpha, \delta}\left(t^{\prime}-s, x-y\right)\right.\right.\right. \\
& \left.\left.\left.\quad-G_{\alpha, \delta}(t-s, x-y)\right)\right]^{2} d y d s\right]^{m / 2} \\
& +C_{m}\left[\int_{t}^{t^{\prime}} \int_{0}^{1}\left(K_{H}^{*} G_{\alpha, \delta}\left(t^{\prime}-s, x^{\prime}-y\right)\right)^{2} d y d s\right]^{m / 2} \\
& =C_{m}(I+I I+I I I) .
\end{aligned}
$$

Using the continuous embedding established in [27]

$$
L^{1 / H}([0, T] \times[0,1]) \subset \mathscr{H},
$$

we obtain

$$
\begin{aligned}
& I=\left[\int _ { 0 } ^ { t } \int _ { 0 } ^ { 1 } \left[K _ { H } ^ { * } \left(G_{\alpha, \delta}\left(t^{\prime}-s, x^{\prime}-y\right)\right.\right.\right. \\
& \left.\left.\left.-G_{\alpha, \delta}\left(t^{\prime}-s, x-y\right)\right)\right]^{2} d y d s\right]^{m / 2} \\
& \leq C_{H}\left[\int _ { 0 } ^ { t } \int _ { 0 } ^ { 1 } \left(G_{\alpha, \delta}\left(t^{\prime}-s, x^{\prime}-y\right)\right.\right. \\
& \left.\left.-G_{\alpha, \delta}\left(t^{\prime}-s, x-y\right)\right)^{1 / H} d y d s\right]^{m H} \\
& =C_{H} \| G_{\alpha, \delta}\left(t^{\prime}-\cdot, x^{\prime}-*\right) \\
& -G_{\alpha, \delta}\left(t^{\prime}-\cdot, x-*\right) \|_{L^{1 / H}([0, T] \times[0,1])}^{m} \\
& =C_{H} \|\left|G_{\alpha, \delta}\left(t^{\prime}-\cdot, x^{\prime}-*\right)-G_{\alpha, \delta}\left(t^{\prime}-\cdot, x-*\right)\right|^{\theta} \\
& \cdot \mid G_{\alpha, \delta}\left(t^{\prime}-\cdot, x^{\prime}-*\right) \\
& -\left.G_{\alpha, \delta}\left(t^{\prime}-\cdot, x-*\right)\right|^{1-\theta} \|_{L^{1 / H}([0, T] \times[0,1])}^{m} \\
& \leq C_{H}\left[\|\left|G_{\alpha, \delta}\left(t^{\prime}-\cdot, x^{\prime}-*\right)-G_{\alpha, \delta}\left(t^{\prime}-\cdot, x-*\right)\right|^{\theta}\right. \\
& \cdot\left|G_{\alpha, \delta}\left(t^{\prime}-\cdot, x^{\prime}-*\right)\right|^{1-\theta} \|_{L^{1 / H}([0, T] \times[0,1])}^{m}
\end{aligned}
$$

$$
\begin{aligned}
& +\|\left|G_{\alpha, \delta}\left(t^{\prime}-\cdot, x^{\prime}-*\right)-G_{\alpha, \delta}\left(t^{\prime}-\cdot, x-*\right)\right|^{\theta} \\
& \left.\cdot\left|G_{\alpha, \delta}\left(t^{\prime}-\cdot, x-*\right)\right|^{1-\theta} \|_{L^{1 / H}([0, T] \times[0,1])}^{m}\right] \\
& =C_{H}\left(I_{1}+I_{2}\right) .
\end{aligned}
$$

Let $\theta \in(0, \min \{1,(\alpha+1) H-1\})$. Thanks to the meanvalue theorem, one can get

$$
\begin{aligned}
I_{1}=||\left|x^{\prime}-x\right|^{\theta}\left|\frac{\partial}{\partial x} G_{\alpha, \delta}\left(t^{\prime}-\cdot, \xi-*\right)\right|^{\theta} \\
\quad \times\left|G_{\alpha, \delta}\left(t^{\prime}-\cdot, x^{\prime}-*\right)\right|^{1-\theta}||_{L^{1 / H}([0, T] \times[0,1])}^{m} \\
=\left(\left|x^{\prime}-x\right|^{\theta / H} \int_{0}^{T} \int_{0}^{1}\left|\frac{\partial}{\partial x} G_{\alpha, \delta}\left(t^{\prime}-s, \xi-y\right)\right|^{\theta / H}\right. \\
\left.\quad \times\left|G_{\alpha, \delta}\left(t^{\prime}-s, x^{\prime}-y\right)\right|^{(1-\theta) / H} d y d s\right)^{m H}, \\
\int_{0}^{1}\left|\frac{\partial}{\partial x} G_{\alpha, \delta}\left(t^{\prime}-s, \xi-y\right)\right|^{\theta / H} \\
\quad \times\left|G_{\alpha, \delta}\left(t^{\prime}-s, x^{\prime}-y\right)\right|^{(1-\theta) / H} d y \\
\leq \int_{\mathbb{R}}\left|\frac{\partial}{\partial x} G_{\alpha, \delta}\left(t^{\prime}-s, \xi-y\right)\right|^{\theta / H} \\
\times\left|G_{\alpha, \delta}\left(t^{\prime}-s, x^{\prime}-y\right)\right|^{(1-\theta) / H} d y \\
=\left(t^{\prime}-s\right)^{1 / \alpha-(1+\theta) / \alpha H} \int_{\mathbb{R}}\left|\frac{\partial}{\partial x} G_{\alpha, \delta}(1, \xi-y)\right|^{\theta / H} \times\left|G_{\alpha, \delta}(1, y)\right|^{(1-\theta) / H} d y
\end{aligned}
$$

Therefore, if $1 / \alpha-(1+\theta) / \alpha H+1>0$, that is, $\theta<(\alpha+1) H-1$, then

$$
I_{1} \leq C_{T, H, \theta}\left|x^{\prime}-x\right|^{m \theta}
$$

Similarly, one can get

$$
I_{2} \leq C_{T, H, \theta}\left|x^{\prime}-x\right|^{m \theta} .
$$

Hence

$$
I \leq C_{T, H, \theta}\left|x^{\prime}-x\right|^{m \theta} .
$$


Now we are in the position to deal with $I I$. Consider

$$
\begin{aligned}
& I I=\left[\int _ { 0 } ^ { t } \int _ { 0 } ^ { 1 } \left[K _ { H } ^ { * } \left(G_{\alpha, \delta}\left(t^{\prime}-s, x-y\right)\right.\right.\right. \\
& \left.\left.\left.-G_{\alpha, \delta}(t-s, x-y)\right)\right]^{2} d y d s\right]^{m / 2} \\
& \leq C_{H}\left[\int _ { 0 } ^ { t } \int _ { 0 } ^ { 1 } \left(G_{\alpha, \delta}\left(t^{\prime}-s, x-y\right)\right.\right. \\
& \left.\left.-G_{\alpha, \delta}(t-s, x-y)\right)^{1 / H} d y d s\right]^{m H} \\
& =C_{H} \| G_{\alpha, \delta}\left(t^{\prime}-\cdot, x-*\right) \\
& -G_{\alpha, \delta}(t-\cdot, x-*) \|_{L^{1 / H}([0, T] \times[0,1])}^{m} \\
& =C_{H} \|\left|G_{\alpha, \delta}\left(t^{\prime}-\cdot, x-*\right)-G_{\alpha, \delta}(t-\cdot, x-*)\right|^{\mu} \\
& \cdot \mid G_{\alpha, \delta}\left(t^{\prime}-\cdot, x-*\right) \\
& -\left.G_{\alpha, \delta}(t-\cdot, x-*)\right|^{1-\mu} \|_{L^{1 / H}([0, T] \times[0,1])}^{m} \\
& \leq C_{H}\left[\|\left|G_{\alpha, \delta}\left(t^{\prime}-\cdot, x-*\right)-G_{\alpha, \delta}(t-\cdot, x-*)\right|^{\mu}\right. \\
& \cdot\left|G_{\alpha, \delta}\left(t^{\prime}-\cdot, x-*\right)\right|^{1-\mu} \|_{L^{1 / H}([0, T] \times[0,1])}^{m} \\
& +\|\left|G_{\alpha, \delta}\left(t^{\prime}-\cdot, x-*\right)-G_{\alpha, \delta}(t-\cdot, x-*)\right|^{\mu} \\
& \left.\cdot\left|G_{\alpha, \delta}(t-\cdot, x-*)\right|^{1-\mu} \|_{L^{1 / H}([0, T] \times[0,1])}^{m}\right] \\
& =C_{H}\left(I I_{1}+I I_{2}\right) .
\end{aligned}
$$

By mean-value theorem, it holds that

$$
\begin{gathered}
I_{1}=||\left|t^{\prime}-t\right|^{\mu}\left|\frac{\partial}{\partial t} G_{\alpha, \delta}(\eta-\cdot, x-*)\right|^{\mu} \\
\cdot\left|G_{\alpha, \delta}\left(t^{\prime}-\cdot, x-*\right)\right|^{1-\mu}||_{L^{1 / H}([0, T] \times[0,1])}^{m} \\
=\left(\int_{0}^{T} \int_{0}^{1}\left|t^{\prime}-t\right|^{\mu / H}\left|\frac{\partial}{\partial t} G_{\alpha, \delta}(\eta-s, x-y)\right|^{\mu / H}\right. \\
\left.\cdot\left|G_{\alpha, \delta}\left(t^{\prime}-s, x-y\right)\right|^{(1-\mu) / H} d y d s\right)^{m H} .
\end{gathered}
$$

Noting that

$$
G_{\alpha, \delta}(t, x)=t^{-1 / \alpha} G_{\alpha, \delta}\left(1, t^{-1 / \alpha} x\right),
$$

one can get

$$
\begin{aligned}
\frac{\partial}{\partial t} G_{\alpha, \delta} & (t, x) \\
= & \left(-\frac{1}{\alpha}\right) t^{-1-1 / \alpha} G_{\alpha, \delta}\left(1, t^{-1 / \alpha} x\right) \\
& +\left(-\frac{1}{\alpha}\right) t^{-1-2 / \alpha}\left[\left.x \frac{\partial}{\partial z} G_{\alpha, \delta}(1, z)\right|_{z=t^{-1 / \alpha} x}\right] .
\end{aligned}
$$

Then

$$
\begin{gathered}
\int_{0}^{1}\left|\frac{\partial}{\partial t} \mathscr{G}_{\alpha, \delta}(\eta-s, x-y)\right|^{\mu / H} \\
\cdot\left|\mathscr{G}_{\alpha, \delta}\left(t^{\prime}-s, x-y\right)\right|^{(1-\mu) / H} d y \\
\leq \int_{R}\left|\frac{\partial}{\partial t} \mathscr{G}_{\alpha, \delta}(\eta-s, x-y)\right|^{\mu / H} \\
\cdot\left|\mathscr{G}_{\alpha, \delta}\left(t^{\prime}-s, x-y\right)\right|^{(1-\mu) / H} d y \\
\leq \int_{R} \mid\left(-\frac{1}{\alpha}\right)(\eta-s)^{-1-1 / \alpha} G_{\alpha, \delta}\left(1,(\eta-s)^{-1 / \alpha}(x-y)\right) \\
\quad+\left(\frac{1}{\alpha}\right)(\eta-2)^{-1-2 / \alpha} \\
\times\left.\left[\left.(x-y) \frac{\partial}{\partial z} G_{\alpha, \delta}(1, z)\right|_{z=(\eta-s)^{-1 / \alpha}(x-y)}\right]\right|^{\mu / H} \\
\cdot\left|\mathscr{G}_{\alpha, \delta}\left(t^{\prime}-s, x-y\right)\right|^{(1-\mu) / H} d y \\
\leq C_{\alpha, \delta, H}\left[(t-s)^{-(\alpha \mu+1) / H \alpha+1 / \alpha}\right. \\
\left.+(t-s)^{-(\alpha \mu+\mu+1) / H \alpha+1 / \alpha}\right] .
\end{gathered}
$$

Therefore, if $\mu \in(0,((\alpha+1) H-1) / \alpha)$, we have

$$
I I_{1} \leq C_{\alpha, \delta, H, T, \mu}\left|t^{\prime}-t\right|^{m \mu}
$$

Similarly,

$$
I I_{2} \leq C_{\alpha, \delta, H, T, \mu}\left|t^{\prime}-t\right|^{m \mu}
$$

Thus, we have

$$
I I \leq C_{T, H, \theta}\left|t^{\prime}-t\right|^{m \mu} .
$$

Now we deal with $I I I$; similar to the proof of $I$, we get

$$
\begin{aligned}
I I I= & {\left[\int_{t}^{t^{\prime}} \int_{0}^{1}\left(K_{H}^{*} G_{\alpha, \delta}\left(t^{\prime}-s, x^{\prime}-y\right)\right)^{2} d y d s\right]^{m / 2} } \\
\leq & C_{\alpha, \delta, H}\left[\int_{t}^{t^{\prime}} \int_{0}^{1}\left|G_{\alpha, \delta}\left(t^{\prime}-s, x^{\prime}-y\right)\right|^{1 / H} d y d s\right]^{m H} \\
\leq & C_{\alpha, \delta, H}\left[\int_{t}^{t^{\prime}}\left(t^{\prime}-t\right)^{-1 / H \alpha+1 / \alpha}\right. \\
& \left.\times\left(\int_{0}^{1}\left|G_{\alpha, \delta}(1, z)\right|^{1 / H} d z\right) d s\right]^{m H} \\
\leq & C_{\alpha, \delta, H}\left|t^{\prime}-t\right|^{m(((\alpha+1) H-1) / \alpha)} .
\end{aligned}
$$

Together with (40)-(55), one can get

$$
\begin{aligned}
& \sup _{n \geq 1} E\left|X_{n}\left(t^{\prime}, x^{\prime}\right)-X_{n}(t, x)\right|^{m} \\
& \quad \leq C\left[\left|t^{\prime}-t\right|^{m \mu}+\left|x^{\prime}-x\right|^{m \theta}\right] .
\end{aligned}
$$

By Lemma 3, the proof can be completed. 
Proposition 6. The family of processes $\left\{X_{n}, n \in \mathbb{N}\right\}$ defined by (33) converges to the process $X$ given by (34), in the sense of finite-dimensional distributions, as $n$ tends to infinity, in the space $\mathscr{C}([0, T] \times[0,1])$.

Proof. We claim that, for any $a_{1}, \ldots, a_{m} \in \mathbb{R}$ and $\left(t_{1}, x_{1}\right), \ldots,\left(t_{m}, x_{m}\right) \in[0, T] \times[0,1]$, the law of linear combination

$$
\sum_{j=1}^{m} a_{j} X_{n}\left(t_{j}, x_{j}\right)
$$

converges weakly to the law of a random variable defined by

$$
\sum_{j=1}^{m} a_{j} X\left(t_{j}, x_{j}\right)
$$

This will be done by proving the convergence of the corresponding characteristic functions; that is,

$$
\begin{aligned}
& E\left[\exp \left\{i \xi \sum_{j=1}^{m} a_{j} X_{n}\left(t_{j}, x_{j}\right)\right\}\right] \\
& \longrightarrow E\left[\exp \left\{i \xi \sum_{j=1}^{m} a_{j} X\left(t_{j}, x_{j}\right)\right\}\right] .
\end{aligned}
$$

Since for any fixed $(t, x) \in[0, T] \times[0,1], K_{H}^{*} G_{\alpha, \delta}(t-s, x-y) \in$ $L^{2}([0, T] \times[0,1])$, then for any $\left(t_{j}, x_{j}\right) \in[0, T] \times[0,1], j \in$ $\{1,2, \ldots, m\}$, there exists a sequence $\left(K_{H}^{*} G_{\alpha, \delta}\left(t_{j}-s, x_{j}-y\right)\right)_{k}$ of simple functions such that $\left(K_{H}^{*} G_{\alpha, \delta}\left(t_{j}-s, x_{j}-y\right)\right)_{k}$ converges to $K_{H}^{*} G_{\alpha, \delta}\left(t_{j}-s, x_{j}-y\right)$ in $L^{2}([0, T] \times[0,1])$ as $k \rightarrow \infty$.

To simplify notation, we define

$$
\begin{gathered}
X_{n}^{j, k}=\int_{0}^{t} \int_{0}^{1}\left(K_{H}^{*} G_{\alpha, \delta}\left(t_{j}-s, x_{j}-y\right)\right)_{k} \theta_{n}(s, y) d y d s, \\
X^{j, k}=\int_{0}^{t} \int_{0}^{1}\left(K_{H}^{*} G_{\alpha, \delta}\left(t_{j}-s, x_{j}-y\right)\right)_{k} W(d s, d y) .
\end{gathered}
$$

Then

$$
\begin{aligned}
& \mid E\left[\exp \left\{i \xi \sum_{j=1}^{m} a_{j} X_{n}\left(t_{j}, x_{j}\right)\right\}\right] \\
& -E\left[\exp \left\{i \xi \sum_{j=1}^{m} a_{j} X\left(t_{j}, x_{j}\right)\right\}\right] \leq V+V I+V I I,
\end{aligned}
$$

where

$$
\begin{array}{r}
V=\mid E\left[\exp \left\{i \xi \sum_{j=1}^{m} a_{j} X_{n}\left(t_{j}, x_{j}\right)\right\}\right] \\
-E\left[\exp \left\{i \xi \sum_{j=1}^{m} a_{j} X_{n}^{j, k}\right\}\right],
\end{array}
$$

$$
\begin{array}{r}
V I=\mid E\left[\exp \left\{i \xi \sum_{j=1}^{m} a_{j} X_{n}^{j, k}\right\}\right] \\
-E\left[\exp \left\{i \xi \sum_{j=1}^{m} a_{j} X^{j, k}\right\}\right], \\
V I I=\mid E\left[\exp \left\{i \xi \sum_{j=1}^{m} a_{j} X^{j, k}\right\}\right] \\
-E\left[\exp \left\{i \xi \sum_{j=1}^{m} a_{j} X\left(t_{j}, x_{j}\right)\right\}\right] .
\end{array}
$$

We will proceed to prove (59) in three steps.

Step 1. By the mean-value theorem, there exists a constant $C>$ 0 such that

$$
\begin{gathered}
V \leq C \max _{1 \leq j \leq m}\left\{E\left|X_{n}\left(t_{j}, x_{j}\right)-X_{n}^{j, k}\right|\right\} \\
=C \max _{1 \leq j \leq m}\left\{E \mid \int_{0}^{T} \int_{0}^{1}\left[K_{H}^{*} G_{\alpha, \delta}\left(t_{j}-s, x_{j}-y\right)\right.\right. \\
\left.-\left(K_{H}^{*} G_{\alpha, \delta}\left(t_{j}-s, x_{j}-y\right)\right)_{k}\right] \\
\left.\times \theta_{n}(s, y) d y d s \mid\right\} .
\end{gathered}
$$

Using the same method as the proof of Lemma 4, by Hölder inequality we can get

$$
\begin{array}{r}
E \mid \int_{0}^{T} \int_{0}^{1}\left[K_{H}^{*} G_{\alpha, \delta}\left(t_{j}-s, x_{j}-y\right)\right. \\
\left.-\left(K_{H}^{*} G_{\alpha, \delta}\left(t_{j}-s, x_{j}-y\right)\right)_{k}\right] \theta_{n}(s, y) d y d s \mid \\
\leq C\left(\int _ { 0 } ^ { T } \int _ { 0 } ^ { 1 } \left[K_{H}^{*} G_{\alpha, \delta}\left(t_{j}-s, x_{j}-y\right)\right.\right. \\
\left.\left.\quad-\left(K_{H}^{*} G_{\alpha, \delta}\left(t_{j}-s, x_{j}-y\right)\right)_{k}\right]^{2} d y d s\right)^{1 / 2} .
\end{array}
$$

So $V$ uniformly converges to 0 with respect to $n$. 
Step 2. We proceed to deal with VI. Using the mean-value theorem again,

$$
\begin{gathered}
V I \leq C \max _{1 \leq j \leq m}\left\{E\left|X_{n}^{j, k}-X^{j, k}\right|\right\} \\
=C \max _{1 \leq j \leq m}\left\{E \mid \int_{0}^{T} \int_{0}^{1}\left(K_{H}^{*} G_{\alpha, \delta}\left(t_{j}-s, x_{j}-y\right)\right)_{k}\right. \\
\quad \times \theta_{n}(s, y) d y d s \\
-\int_{0}^{T} \int_{0}^{1}\left(K_{H}^{*} G_{\alpha, \delta}\left(t_{j}-s, x_{j}-y\right)\right)_{k} \\
\times W(d s, d y) \mid\} .
\end{gathered}
$$

Thanks to Donsker's theorem, as $n \rightarrow \infty$, the laws of processes

$$
\int_{0}^{T} \int_{0}^{1}\left(K_{H}^{*} G_{\alpha, \delta}\left(t_{j}-s, x_{j}-y\right)\right)_{k} \theta_{n}(s, y) d y d s
$$

converge weakly to the law of

$$
\int_{0}^{T} \int_{0}^{1}\left(K_{H}^{*} G_{\alpha, \delta}\left(t_{j}-s, x_{j}-y\right)\right)_{k} W(d s, d y),
$$

since $\left(K_{H}^{*} G_{\alpha, \delta}\left(t_{j}-s, x_{j}-y\right)\right)_{k}$ is a simple function. So we can get $V I \rightarrow 0$, as $n \rightarrow \infty$.

Step 3. Finally we deal with VII. Using the mean-value theorem again, one can get

$$
\begin{gathered}
V I I \leq C \max _{1 \leq j \leq m}\left\{E\left|X^{j, k}-X\left(t_{j}, x_{j}\right)\right|\right\} \\
=C \max _{1 \leq j \leq m}\left\{E \mid \int_{0}^{T} \int_{0}^{1}\left(K_{H}^{*} G_{\alpha, \delta}\left(t_{j}-s, x_{j}-y\right)\right)_{k}\right. \\
\times W(d s, d y) \\
-\int_{0}^{T} \int_{0}^{1} K_{H}^{*} G_{\alpha, \delta}\left(t_{j}-s, x_{j}-y\right) \\
\times W(d s, d y) \mid\} .
\end{gathered}
$$

Then by the Hölder inequality and the variance for a stochastic integral, we get

$$
\begin{gathered}
E \mid \int_{0}^{T} \int_{0}^{1}\left(K_{H}^{*} G_{\alpha, \delta}\left(t_{j}-s, x_{j}-y\right)\right)_{k} W(d s, d y) \\
\quad-\int_{0}^{T} \int_{0}^{1} K_{H}^{*} G_{\alpha, \delta}\left(t_{j}-s, x_{j}-y\right) W(d s, d y) \mid \\
\leq\left(\int _ { 0 } ^ { T } \int _ { 0 } ^ { 1 } \left[\left(K_{H}^{*} G_{\alpha, \delta}\left(t_{j}-s, x_{j}-y\right)\right)_{k}\right.\right. \\
\left.\left.\quad-K_{H}^{*} G_{\alpha, \delta}\left(t_{j}-s, x_{j}-y\right)\right]^{2} d y d s\right)^{1 / 2} .
\end{gathered}
$$

So, VII $\rightarrow 0$, for all $1 \leq j \leq m$, as $n \rightarrow \infty$. Our proof is completed.

As a consequence of the last two properties, we can state the following.

Theorem 7. The family of processes $\left\{X_{n}, n \in \mathbb{N}\right\}$ defined by (33) converges in law, as $n$ tends to infinity, in the space $\mathscr{C}([0, T] \times[0,1])$, to the process $X$ defined by $(34)$.

Now, we can give the proof of Theorem 2.

Proof of Theorem 2. Let us recall first the mild solution of (5), which is given by

$$
\begin{aligned}
u(t, x)= & \int_{0}^{1} G_{\alpha, \delta}(t, x-y) u_{0}(y) d y \\
& +\int_{0}^{t} \int_{0}^{1} G_{\alpha, \delta}(t-s, x-y) f(s, y, u(s, y)) d y d s \\
& +\int_{0}^{t} \int_{0}^{1} K_{H}^{*} G_{\alpha, \delta}(t-s, x-y) W(d s, d y)
\end{aligned}
$$

and the approximation sequence toward the mild solution of (5), which fulfils

$$
\begin{aligned}
u_{n}(t, x)= & \int_{0}^{1} G_{\alpha, \delta}(t, x-y) u_{0}(y) d y \\
& +\int_{0}^{t} \int_{0}^{1} G_{\alpha, \delta}(t-s, x-y) f\left(s, y, u_{n}(s, y)\right) d y d s \\
& +\int_{0}^{t} \int_{0}^{1} K_{H}^{*} G_{\alpha, \delta}(t-s, x-y) \theta_{n}(s, y) d y d s
\end{aligned}
$$

where $\left\{\theta_{n}(t, x),(t, x) \in[0, T] \times[0,1]\right\}, n \in \mathbb{N}$, stand for the Kac-Stroock process which is square integrable a.s.

Moreover, the approximating sequence $u_{n}$ has continuous paths a.s., for all $n \in \mathbb{N}$ which can be obtained by using the properties of the Green function, the fact that $\theta_{n} \in L^{2}([0, T] \times$ $[0,1])$ a.s., together with a Gronwall-type argument.

On the other hand, consider the following function:

$$
\begin{array}{r}
\psi: \mathscr{C}([0, T] \times[0,1]) \longrightarrow \mathscr{C}([0, T] \times[0,1]) \\
\eta \longrightarrow z_{\eta}
\end{array}
$$

where $\eta:[0, T] \times[0,1] \rightarrow \mathbb{R}$ is a continuous function, and

$$
\begin{aligned}
z_{\eta}(t, x)= & \int_{0}^{1} G_{\alpha, \delta}(t, x-y) u_{0}(y) d y \\
& +\int_{0}^{t} \int_{0}^{1} G_{\alpha, \delta}(t-s, x-y) f\left(s, y, u_{n}(s, y)\right) d y d s \\
& +\eta(t, x) .
\end{aligned}
$$


Then it can be proved that this last function admits a unique continuous solution. Now, according to Theorem 3.5 in Bardina et al. [21], the function $\psi$ is continuous. Considering

$$
\begin{gathered}
X_{n}(t, x)=\int_{0}^{t} \int_{0}^{1} K_{H}^{*} G_{\alpha, \delta}(t-s, x-y) \theta_{n}(s, y) d y d s, \\
X(t, x)=\int_{0}^{t} \int_{0}^{1} K_{H}^{*} G_{\alpha, \delta}(t-s, x-y) W(d s, d y),
\end{gathered}
$$

one can get that $X_{n}$ converges in law in $\mathscr{C}([0, T] \times[0,1])$ to $X$, as $n$ goes to infinity. On the other hand, we have that $u_{n}=$ $\psi\left(X_{n}\right)$ and $u=\psi(X)$, and hence the continuity of $\psi$ implies the convergence in law of $u_{n}$ to $u$ in $\mathscr{C}([0, T] \times[0,1])$.

\section{Conflict of Interests}

The authors declare that there is no conflict of interests regarding the publication of this paper.

\section{Acknowledgments}

The authors would like to express their sincere gratitude to the editor and the anonymous referees for their valuable comments and error corrections. Xichao Sun is partially supported by Natural Science Foundation of Anhui Province: Stochastic differential equation driven by fractional noise and its application in finance (no. 1408085QA10), Natural Science Foundation of Anhui Province (no. 1408085QA09), and Key Natural Science Foundation of Anhui Education Commission (no. KJ2013A183). Junfeng Liu is partially supported by Mathematical Tianyuan Foundation of China (no. 11226198).

\section{References}

[1] J. Droniou and C. Imbert, "Fractal first order partial differential equations," Archive for Rational Mechanics and Analysis, vol. 182, pp. 229-261, 2004.

[2] V. V. Uchaikin and V. M. Zolotarev, Chance and Stability, Stable Distributions and Their Applications, Modern Probability and Statistics, 1999.

[3] L. Chen, Y. Chai, R. Wu, J. Sun, and T. Ma, "Cluster synchronization in fractional-order complex dynamical networks," Physics Letters A, vol. 376, no. 35, pp. 2381-2388, 2010.

[4] M. Li, W. Zhao, and C. Cattani, "Delay bound: fractal traffic passes through network servers," Mathematical Problems in Engineering, vol. 2013, Article ID 157636, 15 pages, 2013.

[5] M. Li, "Fractal time series-a tutorial review," Mathematical Problems in Engineering, vol. 2010, Article ID 157264, 26 pages, 2010.

[6] S. Cohen and A. Lindner, "A central limit theorem for the sample autocorrelations of a Lévy driven continuous time moving average process," Journal of Statistical Planning and Inference, vol. 143, no. 8, pp. 1295-1306, 2013.

[7] K. Jaczak-Borkowska, "Generalized BSDEs driven by fractional Brownian motion," Statistics and Probability Letters, vol. 83, no. 3, pp. 805-811, 2013.

[8] M. Besal and C. Rovira, "Stochastic Volterra equations driven by fractional Brownian motion with Hurst parameter $H>1 / 2$," Stochastics and Dynamics, vol. 12, no. 4, pp. 1-24, 2012.
[9] D. T. Nguyen, "Mackey-Glass equation driven by fractional Brownian motion," Physica A, vol. 391, no. 22, pp. 5465-5472, 2012.

[10] Y. S. Mishura and S. V. Posashkova, "Stochastic differential equations driven by a Wiener process and fractional Brownian motion: convergence in Besov space with respect to a parameter," Computers and Mathematics with Applications, vol. 62, no. 3, pp. 1166-1180, 2011.

[11] M. Li and W. Zhao, "Quantitatively investigating locally weak stationarity of modified multifractional Gaussian noise," Physica A, vol. 391, no. 24, pp. 6268-6278, 2012.

[12] M. Li, Y. Q. Chen, J. Y. Li, and W. Zhao, "Hölder scales of sea level," Mathematical Problems in Engineering, vol. 2012, Article ID 863707, 22 pages, 2012.

[13] C. Mueller, “The heat equation with Lévy noise 1," Stochastic Processes and Their Applications, vol. 74, no. 1, pp. 67-82, 1998.

[14] J. Wu, "Fractal Burgers equation with stable Lévy noise," in Proceedings of the 7th International Conference SPDE and Applications, January 2004.

[15] T. Chang and K. Lee, "On a stochastic partial differential equation with a fractional Laplacian operator," Stochastic Processes and Their Applications, vol. 122, no. 9, pp. 3288-3311, 2012.

[16] A. Truman and J. Wu, "Fractal Burgers equation driven by Lévy noises," in Stochastic Partial Differential Equations and Applications, G. da Prato and L. Tubaro, Eds., vol. 245 of Lecture Notes in Pure and Applied Mathematics, pp. 295-310, Chapman Hall/CRC Taylor, 2006.

[17] J. Liu, L. Yan, and Y. Cang, "On a jump-type stochastic fractional partial differential equation with fractional noises," Nonlinear Analysis: Theory, Methods \& Applications, vol. 75, no. 16, pp. 6060-6070, 2012.

[18] D. Wu, "On the solution process for a stochastic fractional partial differential equation driven by space-time white noise," Statistics and Probability Letters, vol. 81, no. 8, pp. 1161-1172, 2011.

[19] M. S. Taqqu, "Weak convergence to fractional brownian motion and to the rosenblatt process," Zeitschrift für Wahrscheinlichkeitstheorie und Verwandte Gebiete, vol. 31, no. 4, pp. 287-302, 1975.

[20] R. Delgado and M. Jolis, "Weak approximation for a class of Gaussian processes," Journal of Applied Probability, vol. 37, no. 2, pp. 400-407, 2000.

[21] X. Bardina, M. Jolis, and L. Quer-Sardanyons, "Weak convergence for the stochastic heat equation driven by Gaussian white noise," Electronic Journal of Probability, vol. 15, pp. 1267-1295, 2010.

[22] B. Boufoussi and S. Hajji, "An approximation result for a quasi-linear stochastic heat equation," Statistics and Probability Letters, vol. 80, no. 17-18, pp. 1369-1377, 2010.

[23] T. E. Mellall and Y. Ouknine, "Weak convergence for qualinear stochastic heat equation driven by a fractional noise with Hurst parameter $H \in((1 / 2), 1)$," Stochastics and Dynamics, vol. 13, no. 3, pp. 1-13, 2013.

[24] L. Debbi, "On some properties of a high order. Fractional differential operator which is not in general selfadjoint," Applied Mathematical Sciences, vol. 1, pp. 1325-1339, 2007.

[25] L. Debbi and M. Dozzi, "On the solutions of nonlinear stochastic fractional partial differential equations in one spatial dimension," Stochastic Processes and Their Applications, vol. 115, no. 11, pp. 1764-1781, 2005.

[26] J. B. Walsh, "An introduction to stochastic partial differential equations," in École d'Été de Probabilités de Saint Flour XIV1984, vol. 1180 of Lecture Notes in Mathematics, pp. 266-439, Springer, Berlin, Germany, 1986. 
[27] D. Nualart and Y. Ouknine, "Regularization of quasilinear heat equation by a fractional noise," Stochastics and Dynamics, vol. 4, no. 2, pp. 201-221, 2004.

[28] T. Komatsu, "On the martingale problem for generators of stable processes with perturbations," Osaka Journal of Mathematics, vol. 21, pp. 113-132, 1984. 


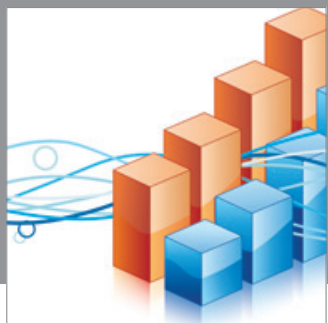

Advances in

Operations Research

mansans

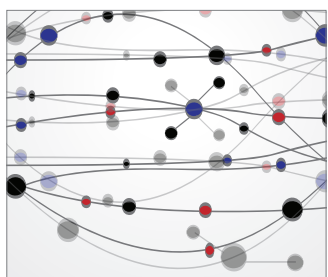

The Scientific World Journal
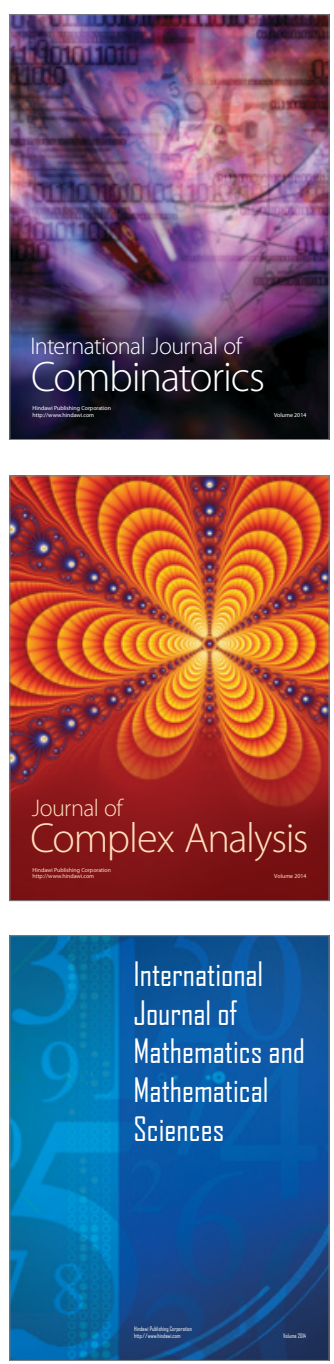
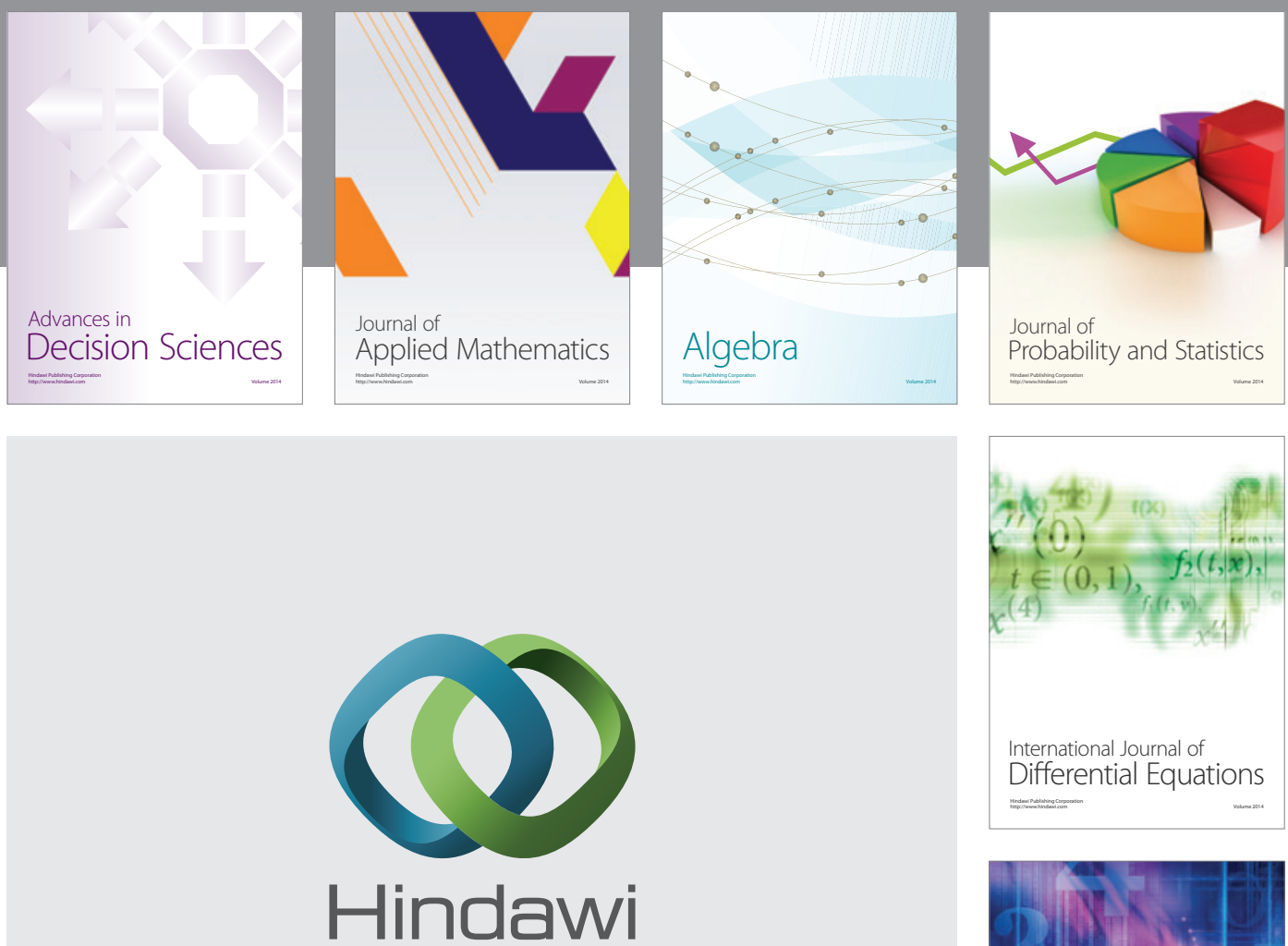

Submit your manuscripts at http://www.hindawi.com
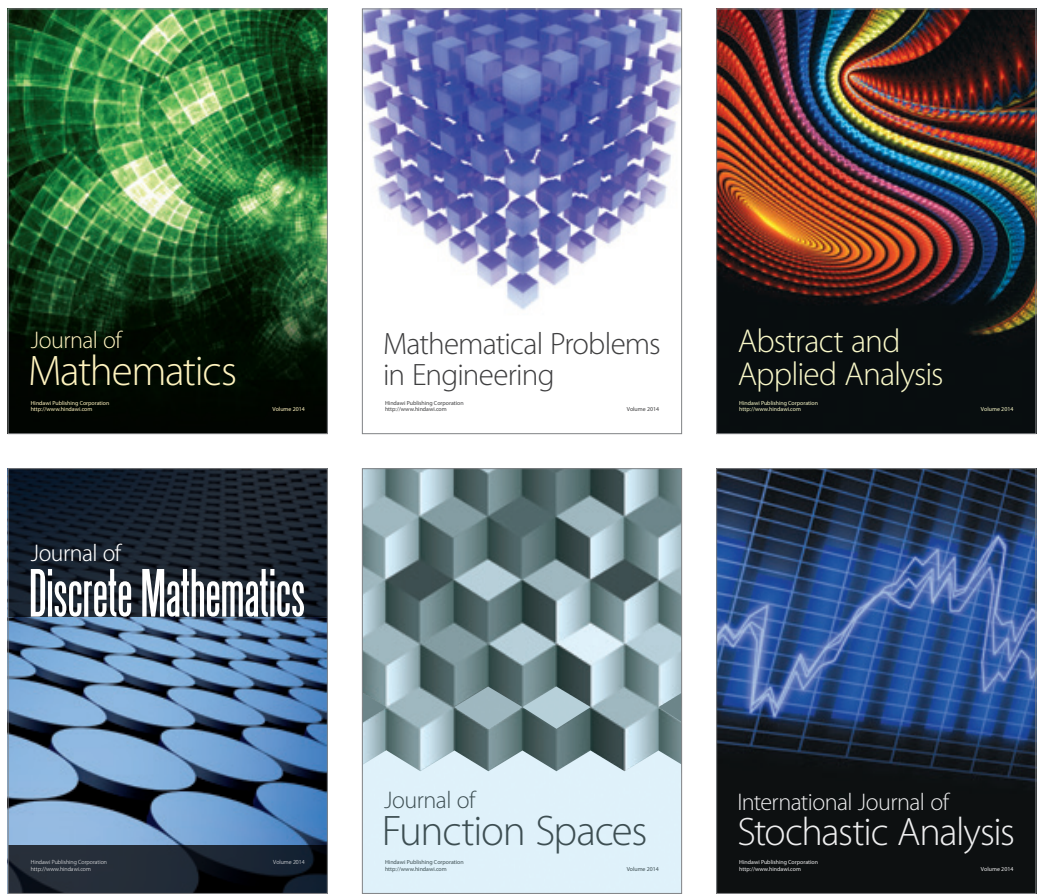

Journal of

Function Spaces

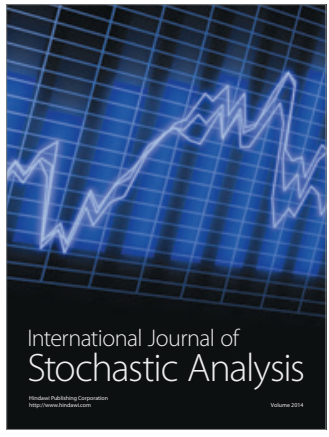

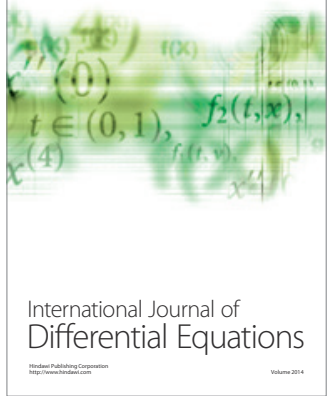
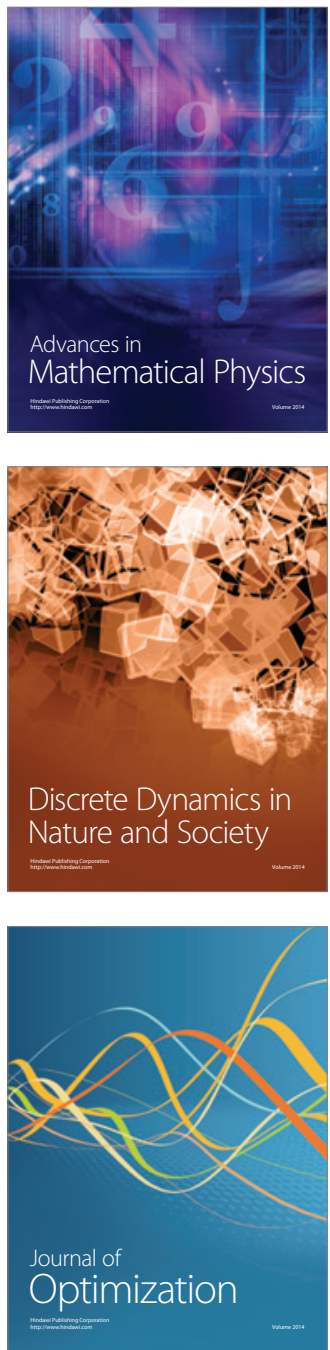